FORMATION Formation emploi

Revue française de sciences sociales

97 | janvier-mars 2007

Former pour dynamiser les territoires

\title{
Mecanic vallée : Interactions entre système productif local et formation
}

"Mecanic vallee": interactions between cluster development and training

Mecanic Vallée: lokale Interaktion zwischen Clusterentwicklung und

Bildungspolitik

Joachim Haas

\section{CpenEdition}

Journals

Édition électronique

URL : http://journals.openedition.org/formationemploi/1653

DOI : 10.4000/formationemploi.1653

ISSN : 2107-0946

Éditeur

La Documentation française

Édition imprimée

Date de publication : 1 janvier 2007

Pagination : $9-21$

ISSN : 0759-6340

\section{Référence électronique}

Joachim Haas, « Mecanic vallée : Interactions entre système productif local et formation », Formation emploi [En ligne], 97 | janvier-mars 2007, mis en ligne le 31 mars 2009, consulté le 30 octobre 2020. URL : http://journals.openedition.org/formationemploi/1653 ; DOI : https://doi.org/10.4000/ formationemploi.1653 


\title{
DOSSIER
}

\section{Mecanic vallée : \\ Interactions entre système productif local et formation}

Par Joachim Haas*

\begin{abstract}
Comment constituer une masse critique d'acteurs engagés afin d'impulser un cercle vertueux de relations entre industrie et appareil de formation? La «Mecanic Vallée » est exemplaire à cet égard.
\end{abstract}

Cet article étudie le renforcement interactif de la politique de formation et du système productif local. Il s'intéresse plus particulièrement aux mécanismes concourant au développement des coopérations interentreprises et entre les sphères publique et privée locales. Le processus mis en exergue suit le principe de la boucle récursive. Selon ce modèle, la progression des coopérations induit des investissements dans le domaine de la formation locale et, inversement, les investissements en matière d'infrastructures de formation font progresser les coopérations. Le projet de système productif local «Mecanic Vallée» est exemplaire à cet égard.

La Délégation à l'aménagement du territoire et à l'action régionale (DATAR) $)^{1}$ a engagé, à la fin des années 90 , une politique d'incitation à la mise en place des réseaux de coopération interentreprises et public/privé. La promotion de ces réseaux - appelés « systèmes productifs locaux (SPL) », « clusters » ou « districts industriels »- s'inspire d'expériences

${ }^{1}$ Aujourd'hui remplacée par la Délégation interministérielle à l'aménagement et à la compétitivité des territoires (DIACT).
* Joachim Haas est sociologue, chargé d'études Céreq au LIRHE (Laboratoire interdisciplinaire de recherche sur les ressources humaines et l'emploil, centre associé au Céreq de Toulouse, université de Toulouse 1. II est diplômé en sociologie et en science politique des universités de Constance, Paris et Heidelberg. Ses recherches portent notamment sur les systèmes d'éducation et d'emploi dans une perspective comparative, sur la sociologie du travail dans l'aéronautique et l'espace, et sur les théories du choix rationnel.

Il a notamment publié : Haas J. (2005), "The Situation in Industry and the Loss of Interest in Science Education", European Journal of Education, 40, 4, pp. 406-416. Haas J. (2006), "Fluctuations des marchés et gestion des ressources humaines dans l'industrie spatiale en France ", in L'évolution de l'emploi et des qualifications dans la construction aéronautique et spatiale», Relief-Céreq, janvier, pp. 147-162. Haas J. (2006), "Occupational Licensing versus Company-led Training. The Controversy over the Competence Assurance System for European Aircraft Technicians", Papier présenté au 16 Congrès mondial de l'Association internationale de sociologie (ISA), Durban, Afrique du Sud, du 23 au 29 juillet 2006. 
réalisées en Italie à partir des années 70. Ces expériences ont montré que la constitution des petites et moyennes entreprises (PME) en cluster améliore notablement leur capacité à affronter le marché mondial (Blanc, 2004). Le cluster se caractérise par la concentration géographique de firmes spécialisées dans un secteur d'activité et engagées dans une dynamique auto-entretenue de coopérations (Porter, 1998).

Susciter de tels réseaux se heurte toutefois à deux obstacles majeurs :

- Un premier obstacle réside dans la non-exclusivité des biens publics ( $c f$. Olson, 1968). Les acquis typiques de la coopération locale - le marché du travail performant, la diffusion de technologies, l'image de marque du bassin, etc. - sont des biens publics, personne ne pouvant être exclu de la jouissance de ces actifs. On peut donc consommer ces biens sans avoir contribué à leur création. Il en découle de fortes tentations opportunistes, dont notamment une incitation aux pratiques du " passager clandestin » cherchant à profiter des acquis collectifs sans régler de cotisation.

- Un second obstacle est lié aux particularités de l'organisation territoriale française. Paulette Pommier, de la DATAR, évoque un éclatement des solidarités traditionnelles locales au cours des décennies de centralisme et de développement exogène (qui privilégie l'arrivée d'investisseurs extérieurs à la région). Ainsi, la politique d'Après-guerre qui a consisté à transférer des établissements industriels du type taylorien de la région parisienne vers les régions plus périphériques aurait concouru «...̀̀ segmenter, à découper, à cloisonner les fonctions et les espaces. Un établissement taylorien n'a pas besoin de relations étroites et complexes d'échanges avec son environnement» (Pommier 2005, p. 9). Parallèlement, un salariat rural se serait développé au détriment d'une dynamique entrepreneuriale endogène à ces zones (Courault, 2000).

Comment réussir à créer un pôle de coopérations dans ce contexte a priori peu porteur pour un tel projet? Cette question constitue la problématique centrale de notre contribution.

La mise en place des coopérations locales sera traitée à l'exemple de l'initiative SPL « Mecanic Vallée ». La Vallée, située dans le sud-ouest du Massif Central, le long de l'arc Tulle-Rodez, comporte environ deux cent petites et moyennes entreprises spécialisées dans la mécanique. Elle figure parmi les 96 projets de SPL sélectionnés par la DATAR. Dans la perspective d'une consolidation des relations, le projet de la Vallée se présente comme une réussite ( $c f$. encadré 1).

Certes, l'inégalité de participation entre les entrepreneurs reste significative. Dans le cas de la Vallée, une dizaine de chefs d'entreprise très actifs constitue le noyau dur et la cheville ouvrière. Une autre catégorie " proactive » est celle des adeptes qui s'efforcent de (faire) respecter une convention sociale qui exige de privilégier l'économie locale. Sont en revanche restés majoritaires les supporteurs passifs et les industriels « invisibles ».

Si l'implication des industriels semble perfectible, la réussite de la Vallée sur le plan relationnel comporte trois aspects :

- La dynamique des activités, auto-entretenue et pilotée par les entrepreneurs. Cette dynamique différencie le projet de la Vallée de maints projets SPL restés dépendants des impulsions des agences intermédiaires ou parapubliques ( $c f$. Pommier, 2005).

- L'implication variée d'acteurs dans la Vallée, représentant les mondes économique, politique et éducatif. La coopération public-privé semble un acquis.

- La coproduction par des acteurs traditionnellement distants. L'appui budgétaire à la Vallée de trois départements et les projets de formation qui associent entreprises et Éducation en constituent des illustrations.

Quels enseignements peut-on tirer de cette réussite? La réponse peut se décliner en trois étapes. La première présente quelques modèles-clés du développement de l'action collective, parmi lesquels se trouve le modèle de l'interaction. Ce modèle, plus spécifiquement l'interaction entre le développement $\mathrm{du}$ cluster et la politique de formation, s'est révélé bien adapté au cas de la «Mecanic Vallée ». La deuxième étape se concentre sur l'analyse des motivations spécifiques des groupes locaux. Elle précise les intérêts qui ont amené les différents acteurs de la Vallée à participer à des actions collectives. La troisième étape porte sur la compréhension des dynamiques. Une série de plusieurs interactions cluster-formation sera traitée en détail. En conclusion, la contribution présente des considérations sur quelques conditionsclés nécessaires au fonctionnement de ce modèle. 


\section{Encadré 1 \\ La « Mecanic Vallée»}

\section{Historique}

1988 à 1992 : politique d'essaimage de firmes de la mécanique par l'entreprise Ratier à Figeac (Lot) ; coopération intensifiée entre Ratier et la municipalité de Figeac louverture d'un Institut universitaire de technologie en 1995).

Années 1990 : actions de parrainage par un club d'industriels du Figeacois pour la qualification de petites et moyennes entreprises locales (élévation de la compétence d'une entreprise sur le plan technologique).

1999 : reconnaissance, par la DATAR (Délégation à l'aménagement du territoire), de cette coopération des firmes comme projet SPL (système productif local). Extension géographique du projet sur l'arc TulleBrive-Figeac-Decazeville-Rodez. Départements concernés: Aveyron, Corrèze, Lot.

En 2000 : création, par les industriels, de l'association « Mecanic Vallée » comme pôle de pilotage. Mise en place de programmes d'actions collectives tournées vers la formation, le transfert de technologies et le développement des marchés. Entrée des organismes locaux de formation dans le mouvement.

\section{Caractéristiques économiques ( $\left.{ }^{*}\right)$}

Les firmes œuvrent principalement pour l'automobile et l'aéronautique en qualité de sous-traitants ou d'équipementiers.

Secteurs concernés (SESSI) : Mécanique générale, fabrication de machines-outils à métaux, fabrication d'équipements automobiles, construction de moteurs pour aéronefs.

Évolution de l'emploi dans ces secteurs sur la période 1993-2001 : 25 \%, sur le territoire de la "Mecanic Vallée », contre $7 \%$ en France.

Potentiel global de ces secteurs sur le territoire en 2004 : 210 entreprises, 14000 emplois, 1,4 milliard d'euros de chiffre d'affaires (CA).

\section{Les acquis entre 1999 et 2004}

Adhérents de l'association « Mecanic Vallée » en 2004: 110 entités dont 81 entreprises de la mécanique, 15 établissements de formation, 10 agences de développement et 4 chambres consulaires. Les 81 entreprises représentent 10200 emplois et 0,9 milliard d'euros de CA.

Infrastructures créées: plusieurs bac pro, brevets de technicien supérieur et licences professionnelles, salon d'affaires annuel spécialisé dans le secteur mécanique.

Relation public-privé : obtention, par l'association «Mecanic Vallée», de subventions conséquentes de la part des instances territoriales. Aisance et fluidité de la coopération Entreprises-Éducation.

Actifs culturels : émergence, parmi les entrepreneurs, d'une convention sociale qui vise à axer davantage les rapports économiques sur la Vallée $\left({ }^{* *}\right)$.

(*) Source : Association « Mecanic Vallée ».

$\left.{ }^{* *}\right)$ : Un industriel témoigne : «Cet esprit qui s'est implanté est important. D’ores et déjà, nombre de contrats inter-entreprises ont été passés; des travaux qui étaient réalisés parfois à l'étranger sont revenus dans la Valée, car les gens ont le sentiment d'appartenir à un club. »(CNER, 2001, p. 59) 


\section{L'INTERDÉPENDANCE DES FACTEURS DE PROGRÈS}

L'essor rapide du projet de cluster « Mecanic Vallée » a motivé notre étude d'exploration des rouages conduisant à cette performance. Les moyens utilisés constituent une série de méthodes qualitatives ( $c f$. encadré 2 ). La période couverte s'étend de 1988 à 2004.

$\mathrm{Au}$ sein de la Vallée se succèdent plusieurs interactions entre le développement du cluster et la politique de formation. L'objectif central de la contribution est d'étayer cette thèse à l'aide d'un retraçage détaillé des processus. Parallèlement, la contribution tend à confirmer l'intégration du modèle de l'interaction cluster-formation au répertoire des facteurs de progrès du cluster.

Précisons les termes utilisés. La notion de progrès ou de développement $d u$ cluster renvoie à l'extension et à l'approfondissement des coopérations locales. La politique de formation renvoie aux programmes des instances privées et publiques qui visent à l'amélioration de la qualification des groupes locaux. Le faisceau de groupes considéré ici est volontairement très large. Il comprend à la fois des groupes formés par des individus (une relève de jeunes, les demandeurs d'emploi...) et des groupes constitués par des entreprises.

Le modèle interaction correspond à des processus d'autorégulation, comme notamment la boucle récursive («feedback loop »). Une des rares applications de ce modèle à l'analyse des systèmes productifs locaux est fournie par le travail de Rosenfeld (2000). Celui-ci a montré le renforcement interactif clusterformation pour quatre bassins situés aux États-Unis et en Europe. Selon la séquence-type constatée, le processus peut commencer par le progrès (institutionnel, associatif) dans le système local; ce progrès incite à son tour les organismes de formation à s'investir, ce qui encourage de nouvelles initiatives institutionnelles et/ou associatives - le cluster progresse.

Le modèle d'interaction est utilisé par un courant scientifique qui tend à répertorier des processus-type d'actions collectives. Les travaux visent notamment l'enrichissement d'un inventaire de modèles qui traitent de la participation à des activités d'intérêt commun dans des configurations qui sont a priori

\section{Encadré 2}

\section{Méthodologie}

Trois outils méthodologiques ont été mobilisés :

- L'exploitation de bases de données, en particulier les archives de la presse régionale, les documents de l'Association «Mecanic Vallée » et les publications des agences de développement. On a ainsi établi une chronologie politique et économique de la Vallée sur une longue période (1988 - 2004), ce qui a permis de mettre à jour une série $d^{\prime}$ interactions cluster-formation.

- Une analyse secondaire de monographies sur les évolutions récentes dans la Vallée. Les monographies traitaient de la politique de formation (Combes et Quenson, 2002) et des relations interentreprises (Dauty, 2003 ; Guillaume, 2003). La comparaison de ces sources révèle que les actions communes de formation densifient en effet la relation entre entreprises et Éducation, mais pas celle entre firmes.

- Trois entretiens semi-directifs, d'une durée de deux heures chacun. Ces échanges ont permis une compréhension plus fine des acteurs centraux de la Vallée. L'entretien avec un cadre d'entreprise a porté sur les stratégies économiques et politiques de Ratier, entreprise initiatrice du mouvement des firmes pour la création d'un SPL (système productif locall. Le thème de l'entretien avec un responsable de formation concernait la réponse des instances éducatives au projet de cluster. Un dernier entretien, mené auprès d'un représentant de la presse, visait à cerner les enjeux des bassins et collectivités territoriales impliqués dans ce projet. Ces entretiens ont complété de nombreux échanges avec les sous-traitants de l'aérospatiale régionale.

dissuasives par rapport à un tel engagement. Ces configurations sont caractérisées par l'hétérogénéité, l'opportunisme des acteurs et l'absence de pouvoir 
dominant. Selon plusieurs modèles de cet inventaire, l'augmentation de la certitude par rapport au résultat est une condition-clé pour susciter l'engagement ( $c f$. Coleman, 1990). Ont été répertoriés, entre autres, pour les analyses de la coopération locale, un certain nombre de rôles, de stratégies et de dynamiques structurelles qui concourent à cette hausse de certitude (et/ou à la baisse du risque de mauvais investissement et exploitation). Parmi les rôles, on compte par exemple le «leader» charismatique; du point de vue de l'acteur, le leader renforce la certitude du résultat à la fois par sa capacité de coordination et par sa dépendance à l'égard de l'assentiment du collectif. Concernant les stratégies, on peut évoquer le modèle de la «confiance conditionnelle» (Lorenz, 1988). Selon ce modèle, l'acteur renforce la certitude du résultat par une réciprocité prompte et démonstrative qui incite son interlocuteur à coopérer : la défection de ce dernier sera sanctionnée et sa coopération sera récompensée. Chaque nouvelle étape est donc l'occasion d'effectuer un test susceptible de remettre en cause ou de prolonger l'engagement.

Parmi les dynamiques structurelles figure le modèle d'interaction évoqué plus haut. Ces dynamiques correspondent à l'image de l'ouverture ou de l'élargissement d'une brèche : le progrès réalisé par un groupe de protagonistes se solde par une croissance des participations en raison de la baisse des coûts d'adhésion et/ou de la hausse des chances de réussite. On peut mentionner comme exemples les modèles du «seuil» (Granovetter, 1978; Macy, 1991) et de la «masse critique » (Oliver, Marwell et Teixeira, 1985). Selon ces modèles, les acquis intermédiaires d'un groupe d'actifs tendent à s'approcher du résultat désiré de telle manière qu'une autre catégorie, plus exigeante par rapport à la certitude du résultat, adhère au mouvement. Dans ces cas, la quantité ou la qualité des acquis intermédiaires (infrastructures, adhésions, alliances, reconnaissance, etc.) constituent une «masse » suffisamment importante pour qu'un autre groupe, plus réservé, accepte d'y participer.

Un point fort des modèles de dynamiques structurelles est qu'elles sont complémentaires à l'approche «factorielle» classique. Celle-ci est par exemple utilisée par Pommier (2005) dans le cadre de son évaluation de la politique SPL de la DATAR. Son analyse retient quatre facteurs-clés pour le succès d'un projet SPL: l'engagement fort de quelques chefs d'entreprise, la volonté stratégique des acteurs publics locaux, l'existence d'un pôle d'animation reconnu et l'implication d'un nombre d'entreprises significatif. Comme on le verra plus loin, l'expérience de la "Mecanic Vallée» confirme l'importance de ces facteurs. La valeur ajoutée des dynamiques structurelles réside dans la mise en évidence de l'interdépendance de ces facteurs. Notre analyse va par exemple montrer que l'engagement des chefs d'entreprise interagit avec la volonté stratégique des acteurs publics, et que cette volonté est en interdépendance avec la performance du pôle d'animation, etc.

\section{LES ACTEURS LOCAUX CONVERGENT VERS L'OBJECTIF CLUSTER}

Trois intérêts majeurs se conjuguent pour densifier la Vallée : une grande firme cherchait à développer un bassin de sous-traitance afin de conforter sa mutation stratégique; les collectivités locales visaient le renforcement d'un pôle industriel pour faire face aux grandes agglomérations; les entreprises de la filière mécanique souhaitaient quant à elles aménager la relation formation-emploi afin d'améliorer l'offre sur le marché professionnel local.

\section{Une sous-traitance de proximité comme support de la mutation stratégique}

L'entreprise Ratier, située à Figeac, a été la «force motrice» du mouvement des entreprises pour la Vallée. On retrouve la firme au cœur des opérations à l'origine des engagements. Son PDG, Robert Vitrat personnalité très charismatique - a pendant longtemps joué le rôle de porte-parole et d'animateur des entrepreneurs.

Ratier Figeac construit des pièces et des équipements aéronautiques pour la fabrication d'hélices, de commandes de cockpit, de vérins de puissance et de modules hydrauliques. L'entreprise, maintenant centenaire, emploie environ un millier de collaborateurs. 
Dès la fin des années 80, la firme engage une mutation stratégique. Elle cherche à abandonner le statut de sous-traitant (exécutant-producteur) pour devenir systémier (concepteur-assembleur). La mutation s'explique par la nécessité de rechercher une position d'excellence technologique moins sensible à une concurrence par les prix.

L'entreprise étendue devient le modèle de cette réorganisation industrielle. Cette configuration se caractérise par la forte externalisation des travaux et l'imbrication des relations avec les repreneurs des tâches cédées. L'externalisation se justifie par le recentrage de la firme sur le «cœur de métier » afin d'honorer les nouvelles exigences en termes d'excellence technique. L'imbrication reflète quant à elle la volonté d'instaurer une relation durable dans laquelle les sous-traitants bénéficient de contrats de longue durée et d'appui technique en contrepartie de la prise en charge des fonctions critiques abandonnées par Ratier. Parmi les fonctions cédées à un nombre croissant de sous-traitants, on compte par exemple l'assurance-qualité des pièces. Dès lors, Ratier ne contrôle plus les produits livrés. Un autre exemple est l'achat de programmes complets, donc la responsabilisation du sous-traitant depuis la conception de la pièce jusqu'à la gestion des stocks à flux tendus.

Au cours des années 90, la firme a développé des relations imbriquées avec une quinzaine de PME situées dans la Vallée. On le verra plus loin, le développement de ce segment local a été capital pour le démarrage du cluster. Deux explications à ce choix peuvent être avancées :

- La première ressort des relations commerciales des PME locales liées à Ratier. La Vallée les alimente à travers un socle de clients et, parallèlement, elles passent des commandes au niveau local. Ainsi, en choisissant un segment de proximité, Ratier recherche un effet stabilisateur des circuits locaux sur ses sous-traitants. L'idée principale du programme SPL de la DATAR, la stimulation de la croissance de ces circuits, correspondait ainsi idéalement à la préoccupation de la firme.

- Un second argument renvoie au type d'activités cédées par Ratier au cours de sa transformation. Celles-ci sont très complexes, et ne permettent pas, $a$ priori, une structuration claire et stable des processus de fabrication. La préparation de ces pièces en soustraitance exige des échanges permanents entre preneur et donneur d'ordre: spécification conjointe, essais, étude de process, monitoring, amélioration continue, input de connaissances tacites. La proximité facilite ce processus ( $c f$. Maskell et Malmberg, 1999). La proximité a donc été recherchée pour conserver leur fluidité aux transactions complexes.

En somme, l'entrée d'une grande firme dans le programme de «l'entreprise étendue » constituait un premier motif de l'émergence de la Vallée. Le concept comporte un volet de sous-traitance de proximité qui porte en germes des avantages relatifs : la stabilité économique des fournisseurs et la fluidité des transactions non standardisées. Ainsi, la proximité peut devenir une ressource recherchée pour sécuriser un repositionnement stratégique complexe ( $c f$. Mohrman, 2003).

\section{Un pôle industriel pour rivaliser avec les grandes agglomérations}

Le périmètre géographique des bassins constituant la Vallée a été conçu et décidé par les firmes à la fin des années 90. L'idée était de dépasser l'îlot pionnier du Figeacois afin de «faire masse» vis-à-vis de l'extérieur et de " faire chaîne intégrée » en interne. Chaque chaînon du cordon ainsi tissé est caractérisé par des enjeux spécifiques : reconversion des firmes liées à la fabrication d'armes (bassin de Tulle), regroupement des PME pour accéder aux contrats plus importants (Brive), pérennisation de la filière aéronautique dans un contexte de forte fluctuation du marché (Figeac), reconversion d'un bassin spécialisé dans la sidéro-métallurgie (Decazeville), rattrapage du déficit structurel d'activités industrielles (Rodez). Préoccupées par des enjeux spécifiques, et dépourvues de liens historiques affirmés, ces zones sont $a$ priori déconnectées, voire rivales. Pourtant, un facteur fort les unit: celui de la menace créée par l'attraction des grandes agglomérations qui joue au détriment des territoires excentrés et semi-ruraux. Le problème commun aux petits bassins de la Vallée est la captation des ressources par les zones métropolitaines du sud-ouest (Bordeaux, Limoges, Toulouse). La zone toulousaine, par exemple, attire $80 \%$ des projets industriels en Midi-Pyrénées².

${ }^{2}$ La Dépêche du Midi, 20/03/2002. 
La perte relative ou absolue de ressources est une conséquence de la "croissance en archipel» survenue dans les années 80 , qui remplace l'ancienne tendance de «l'usine à la campagne » (Veltz, 2002). $\mathrm{Ce}$ nouveau type de croissance redirige les ressources vers les grandes agglomérations hors Îlede-France, au détriment des petits bassins industriels. Selon Veltz, ce changement dans la localisation des activités serait principalement dû à l'importance accrue de la sous-traitance de spécialité et des services aux entreprises, activités favorisées par le brassage métropolitain.

Lémergence de la Vallée s'expliquait donc aussi par l'intérêt, notamment des collectivités locales, pour l'établissement d'un pôle industriel suffisamment fort pour faire face à l'attraction des grandes agglomérations.

\section{Un dispositif local de formation apte à capter la main-d'œuvre juvénile}

Les opérations de formation figuraient parmi les premiers chantiers du mouvement pour un système productif local. La priorité accordée à ces opérations met à jour une autre raison au cœur de l'initiative pour la Vallée. Il s'agit de l'intérêt revendiqué par les entreprises pour une meilleure régulation de l'offre sur le marché professionnel local. Selon de nombreux discours publics, les firmes souffrent de difficultés récurrentes de recrutement et du turnover endémique des personnels. Des aménagements étaient donc nécessaires pour mieux attirer et retenir une main-d'œuvre jeune vers les métiers de la métallurgie/mécanique.

Les causes des difficultés sont multiples. Nous pensons tout d'abord à la spécialisation de la Vallée sur les deux marchés volatils que sont l'aéronautique et l'automobile. La hausse conjoncturelle de ces industries provoque, dans des bassins de sous-traitance tels que la Vallée, des engorgements soudains sur le marché du travail ; la baisse conjoncturelle conduit à une raréfaction de la main-d'œuvre à moyen terme, principalement induite par la désertion ou l'abandon des jeunes du métier et/ou de la localité.

Un autre facteur capital est constitué par la désaffection des jeunes pour les formations de la mécanique. Jusqu'à la fin des années 90 , cette désaffection concernait tous les niveaux de la filière présents dans la Vallée.
L'effondrement du BEP mécanique (productique mécanique option « usinage, outillage, chaudronnerie»), vivier du Bac pro, suscitait de vives inquiétudes.

Enfin, une dernière mais importante source des difficultés de recrutement est la mobilité due à la poursuite d'études. Les départs pour suivre des études non proposées localement constituent généralement des pertes pour le territoire puisque les partants reviennent rarement. Cet effet intervient notamment pour les post-bac et post « bac $+2 »$.

\section{LES INTÉRACTIONS «CLUSTER-FORMATION 》 DENSIFIENT LA VALLÉE}

Selon nos investigations, l'apparition de plusieurs interactions cluster-formation est à la base de l'évolution remarquable de la Vallée. Ces interactions reposent sur l'implantation d'un IUT (Institut universitaire de technologie), sur le parrainage pour la qualification des firmes, sur la valorisation de la filière professionnelle et la diffusion des savoirs techniques.

L'analyse d'une interaction distinguera trois phases : un avancement pro-cluster spécifique, à savoir des progrès sur le plan des coopérations; l'influence de ces progrès sur les politiques locales de formation/ qualification ; les répercussions de ces politiques sur l'évolution du cluster.

\section{Une coalition public/privé soudée}

Lorigine du mouvement pour la Vallée repose sur une alliance politique dont les processus se sont déroulés de 1988 à $1992^{3}$. Cet échange liait deux acteurs du Figeacois: l'entreprise Ratier, en la personne de Robert Vitrat, et la mairie de Figeac, représentée par Martin Malvy. Ratier a contribué à la création d'une colonie d'entreprises de la mécanique par le biais d'une politique d'essaimage, et la municipalité s'est battue pour créer un établissement de formation technique, l'Institut universitaire de technologie de Figeac.

${ }^{3}$ On considère cet événement comme le début du processus, ce qui se justifie dans la mesure où il s'agissait de la première opération privée comportant explicitement une vision de développement industriel du bassin. 
La politique d'essaimage de Ratier résulte de son adoption d'une stratégie «d'entreprise étendue ». À compter de 1987, son carnet de commandes s'accroit fortement; il fallait honorer les commandes par la mobilisation de sous-traitants. Plutôt que d'aller les chercher ailleurs, Ratier a choisi de les créer à ses côtés. En quatre ans, la firme a essaimé, par voie d'externalisation, onze entreprises de sous-traitance de proximité. Elle a recherché et encouragé des porteurs de projets en interne, notamment parmi ses ingénieurs et cadres techniques. Elle les a dotés de moyens pour une réussite quasiment assurée 4 et a honoré la totalité du carnet de commandes de démarrage. Aucun dépôt de bilan n'est intervenu et la colonie essaimée compte aujourd'hui plus de 400 emplois sur le seul bassin de Figeac.

La collectivité locale, quant à elle, s'est impliquée par une campagne de lobbying acharnée pour l'installation d'un IUT. Cette démarche relevait d'une logique de réciprocité vis-à-vis de Ratier - la firme étant le leader d'un dossier IUT déposé par dix-sept entreprises du Lot - tout en permettant de créer une coalition avec les firmes locales pour promouvoir la construction d'un pôle industriel attractif.

La résistance de l'académie de Midi-Pyrénées contre le projet IUT était forte. Martin Malvy témoigne :

« Je me souviens du jour ou, poussé dans le dos par Robert Vitrat et d'autres, j'ai proposé la candidature de Figeac pour un IUT, les sourires ont été grands, $i c i$, en Midi-Pyrénées. Lorsqu'on a voulu faire comprendre qu'il y avait là un réseau d'entreprises de la mécanique, on nous a pratiquement ri au nez! On connaissait Ratier; on commençait à connaître Bosch à Rodez. Personne ne connaissait les autres ou presque pas. » (CNER, 2001, pp. 67-68)

Le défi paraissait d'autant plus difficile à relever qu'il s'agissait d'une implantation universitaire dans une petite ville de 10000 habitants, même pas préfecture de département. Le pari-gagné malgré ces difficultés - suscite donc l'émergence d'un «mythe fondateur» ( $c f$. Raveyre et Saglio, 1984). Selon certains, le succès repose sur l'invitation du

${ }^{4}$ Prêt de l'ensemble des outillages, formation du personnel recruté, aide à la construction des locaux.
Recteur à visiter une journée entière les entreprises du Figeacois («Le soir, il était convaincu... »). D'autres prétendent que le coup décisif a été porté par la menace lancée par Martin Malvy de fermer la maternité locale, menace exprimée après qu'on lui ait rétorqué que les enseignants ne viendraient jamais dans ce coin de terroir. Le premier département de l'IUT est le Génie mécanique, opérationnel depuis 1995.

La chronique des événements liée à l'obtention de l'IUT correspond bien au modèle d'interaction cluster-formation proposé. En amont du projet IUT, il y avait la détermination d'une grande entreprise pour l'instauration d'un pôle industriel local. Cette détermination s'est manifestée par une politique volontariste d'essaimage. Le projet IUT s'inscrit ensuite comme réponse constructive témoignant d'une volonté stratégique également exceptionnelle. En aval de ce projet, les retombées constituent des facteurs importants pour promouvoir la construction d'un cluster. Il s'agit premièrement de la création d'une coalition public/privé soudée par la complicité entre deux personnalités managériales. Deuxièmement, la réussite du projet IUT a ouvert une brèche et révélé «la plasticité des choses ». Ainsi, une autre impulsion à la densification de la Vallée, la mise en place d'une Table Ronde de firmes mécènes, a suivi.

La présentation de cette première interaction clusterformation nous est utile pour souligner une précondition fondamentale du modèle : les questions de formation doivent être centrales pour les acteurs. Cette focalisation résulte typiquement des déficits chroniques dans la structure des ressources humaines du collectif local. Toutefois, dans ce cas, l'investissement des divers acteurs dans la formation n'a pas uniquement comme fonction l'ingénierie de la relation formation-emploi, mais a surtout valeur de symbole. L'investissement exprime l'engagement sérieux de l'acteur pour le développement local. Dans l'exemple de l'IUT, cet engagement se lit au travers des difficultés maîtrisées. En effet, convaincre le Rectorat de lancer un projet risqué témoigne d'une réelle détermination. 


\section{Le mécénat}

pour améliorer les compétences technologiques des PME

Ratier regroupait autour d'une Table Ronde les start$u p$ fraîchement essaimées et quelques petits équipementiers locaux, l'adhésion au cercle étant assurée par les obligations de loyauté (contrat moral vis-à-vis de la firme mère, interconnaissances d'individus passés par les mêmes entreprises ou les mêmes cycles de formation) auxquelles se conjuguait la dépendance économique. La vocation du cercle était la qualification des firmes de la filière mécanique locale. Par «qualification», nous entendons ici l'élévation de la compétence d'une entreprise sur le plan technologique.

«Storm» et «Chassint» sont deux opérations d'appui exemplaires. La «Storm» est une machine d'usinage à grande vitesse (UGV) construite pour le traitement de pièces aéronautiques aux dimensions exceptionnelles. Elle a été conçue et réalisée par cinq entreprises du Figeacois du secteur aéronautique. « Chassint » était un petit carrossier automobile figeacois jusqu'à ce que la Table Ronde ait découvert les capacités de son chef d'entreprise. Grâce à l'appui du cercle, elle a créé un atelier qui emploie aujourd'hui vingt-cinq salariés spécialisés en peinture aéronautique et autres traitements à haute technicité.

Le support commercial, la certification qualité et la transmission programmée des savoirs constituaient les leviers mobilisés pour la qualification des entités sélectionnées. Le support commercial a été appliqué pour le projet «Storm». En se proposant comme premier client et donc comme banc d'essai, Ratier s'était investi avec la volonté affichée de pérenniser la compétence UGV dans la région. La certification qualité comme moyen de qualification a été proposée à certains sous-traitants de spécialité. Sur les conseils techniques de la Table Ronde, ces entreprises ont obtenu des agréments très sélectifs de l'aéronautique, des autorisations pour l'approvisionnement en matières premières, et des certifications de la série ISO 9000. Afin d'obtenir des agréments, « Chassint » par exemple, a introduit une technique de décapage sélectif non polluante ; la Table Ronde s'est chargée de l'étude du process, et la PME l'a mis en œuvre. Les programmes de transmission des savoirs techniques, quant à eux, ont été dispensés aux porteurs de projets comme par exemple les PME essaimées. Ces programmes ont notamment visé «l'informatisation » des entreprises. Il s'agit de formations sur le calcul de structure, sur la conception/fabrication assistée par ordinateur (CAO, FAO), sur la gestion de réseau informatique.

Une seconde interaction cluster-formation se dessine alors. Le processus vers un cluster se confirmait par l'installation d'un IUT et la création d'une coalition public/privé soudée. Ceci a encouragé la constitution d'un «club» de firmes mécènes qui se lançait dans une campagne de qualification des PME locales. Cette activité de formation, à caractère à la fois utile et symbolique, renforçait à son tour le cheminement pro-cluster. Ce cheminement apparaît à travers la reconnaissance publique du cercle de firmes mécènes : convaincu par la performance de ce groupe, un consortium d'agences et de chambres des trois départements Aveyron, Corrèze et Lot s'est constitué, en 1998, pour proposer l'entrée du cercle dans le nouveau programme SPL de la DATAR. Il s'agissait d'obtenir des budgets pour une extension du périmètre conceptuel, organisationnel et géographique du réseau d'entreprises. L'accès à ce programme (1999) et la création de l'association Mecanic Vallée (2000) constituent l'entrée dans la phase d'institutionnalisation du mouvement pro-cluster.

\section{Asșocier entreprises et Éducation pour valoriser la filière professionnelle}

La première grande opération de l'association « Mecanic Vallée » visait l'ajout d'un nouvel élément au système local de formation professionnelle. 24 entreprises s'étaient associées à une démarche de création de sections d'apprentissage conduisant au bac professionnel Productique-Mécanique option « usinage» (PMOU). En 1999, quatre sections PMOU, totalisant une soixantaine de places, ont été installées dans les lycées professionnels de Figeac et Rodez ${ }^{5}$. Ce dispositif a ensuite été complété par une multitude d'autres enseignements techniques en alternance (cf. infra).

${ }^{5}$ Plus une section au Centre de formation d'apprentis de l'industrie de Tulle. 
Ces projets de formation ont-ils contribué à densifier le tissu des coopérations locales? Une répercussion de l'approche collective de la formation sur les comportements des firmes ne nous paraît pas systématique. Ce constat peut être illustré par l'exemple de la gestion des compétences de la Vallée comme une dimension voisine de la politique de formation. Bien que toutes les entreprises de taille importante de la Vallée participent aux projets de formation des jeunes (actions de valorisation de la filière professionnelle, démarches de création de sections) une nette polarisation persiste entre elles sur le plan de la qualification des PME. Selon nos recherches, il existe un clivage entre deux catégories de (grandes) firmes de la Vallée. L'une des catégories est constituée par des fabricants de pièces en grandes séries. $\mathrm{Ce}$ sont des membres passifs de l'association «Mecanic Vallée »; ils engagent très peu de soustraitants industriels locaux et ne participent pas aux initiatives de qualification des PME. L'autre catégorie est caractérisée par les fabricants de systèmes mécaniques complexes en petites séries ou « sur-mesure ». Ces firmes s'engagent activement dans le projet de la Vallée, disposent d'un socle conséquent de sous-traitants locaux et œuvrent pour une montée en qualification des PME.

Si les retombées de l'approche collective de formation sur des relations interentreprises paraissent pour l'instant limitées et fragiles, celles qui relient entreprises et Éducation se sont renforcées. Nous assistons à une fluidité des coopérations qui montre le rapprochement certain entre ces deux milieux souvent cloisonnés et distants ( $c f$. Jobert, 2000). Leur coproduction est devenue très fluide et variée. Pour la filière de la mécanique, on compte, après six ans de travaux en commun (1999 à 2004), la mise en place de quatre sections de bac pro en apprentissage, d'un BTS (brevet de technicien supérieur) en apprentissage et de trois licences professionnelles. Les entreprises de la mécanique et les représentants de l'Éducation travaillent par ailleurs ensemble pour préparer une université multipolaire (intégrant la Vallée) et deux écoles d'ingénieurs (localisées dans la Vallée).

La désaffection pour les métiers techniques ne menace pas uniquement les entreprises. Les acteurs locaux de l'Éducation nationale (proviseurs, chefs de travaux, équipes éducatives de la Vallée) sont également concernés, par le biais des suppressions de sections et de postes. L'histoire des fermetures et des concurrences illustre la réalité de ce risque. Les sections des lycées professionnels de la Vallée sont souvent de petite taille et risquent de ce fait d'être rapidement affectées par un changement des flux démographiques ou scolaires. Ainsi, quatre sections BEP de mécanique ont été supprimées entre 1990 et 2004. La tension liée à la concurrence a augmenté en parallèle. À partir de l'an 2000, les ouvertures de nouvelles sections doivent être financées par des fermetures en région.

L'engagement de firmes pour la Vallée représente par conséquent un potentiel intéressant pour les acteurs locaux de l'Éducation. Le développement de l'industrie conduira en effet à la croissance démographique, scolaire et professionnelle du district et promet donc d'attirer et de retenir plus d'élèves pour les formations correspondantes.

La valorisation de la filière représente un intérêt commun auquel s'ajoute la complémentarité des ressources spécifiques de l'Éducation et de l'entreprise. Ainsi, la capacité d'allocation des enseignements constitue une ressource spécifique du système éducatif qui peut être mobilisée en ce sens. La création d'un bac pro, d'un BTS, d'un DUT (diplôme universitaire de technologie), d'une licence professionnelle sont porteurs d'effets d'attraction et de rétention pour le marché professionnel local. Quant aux entreprises locales, leurs ressources spécifiques pour accroître l'attractivité de la profession résident principalement dans le développement industriel.

Les observations sur les engagements pour la valorisation de la filière professionnelle nous permettent d'exposer une autre interaction cluster-formation. Le mouvement des firmes pour la Vallée a été reconnu publiquement et a pu s'institutionnaliser grâce à son efficience et à sa volonté de pérennité. Un grand chantier prometteur d'une bonne croissance industrielle s'était donc établi. Mais la dynamique risquait de se heurter aux déficits de main-d'œuvre qualifiée. Afin de conforter leur propre situation, les acteurs locaux de l'Éducation ont apporté des ressources complémentaires pour une meilleure régulation de l'offre locale des qualifications. Ce processus a entraîné le rapprochement des milieux économiques 
et éducatifs. Ce rapprochement s'observe à travers la fluidité et la cohérence de leur corégulation de la relation formation-emploi. On assiste donc à l'émergence d'une composante des clusters que Amin et Thrift (1994) qualifient de «la richesse en concertations formelles et informelles » (« institutional thickness »).

\section{L'Éducation nationale diffuse les technologies au plan local}

L'Éducation nationale a concouru au développement local en devenant un centre de transfert de technologies. l'IUT de Figeac en est l'exemple pivot. Cette institution s'est dotée, en 2003, de la machine «Skymill», un centre d'Usinage à Très Grande Vitesse (UTGV) réalisé et industrialisé par un consortium de trois entreprises de la Vallée. Son achat par l'IUT s'est accompagné de la signature d'une charte stipulant que la machine sera accessible aux entreprises locales pour qu'elles se familiarisent aux fonctionnalités de l'UTGV.

La charte vise à faciliter la diffusion locale de la technologie UTGV. L'évolution du marché fait pression sur les PME pour qu'elles l'adoptent ; mais l'investissement (350 000 euros), trois fois plus cher que le conventionnel, n'est rentable qu'à condition que l'ensemble du procédé soit rapidement maîtrisé. Ceci exige une formation intensive du personnel tant à la conduite de la machine qu'à la compréhension de conditions de coupe inédites.

Les freins à l'adoption d'une nouvelle technologie ne se limitent pas aux coûts cumulatifs d'acquisition et de formation. Un autre obstacle réside dans les mentalités typiques aux PME : leur origine et leur petite taille les amènent souvent à privilégier une stratégie purement commerciale pour le développement des marchés ; la veille technologique et l'intégration d'innovations comme moyens de développer les marchés ne constituent que très rarement un axe stratégique établi. Ce phénomène constitue une carence répandue dont les stratèges de la Vallée se sont préoccupés. Après la campagne pour la valorisation de la voie professionnelle, la diffusion des technologies est devenue l'objectif premier de la Vallée.

Les acteurs locaux de l'Éducation auraient pu se comporter de manière traditionnelle en optant pour des technologies éprouvées. L'IUT pouvait acquérir, pour le même prix que la Skymill, une gamme d'outils pédagogiques - un centre d'usinage, un centre de tournage et une électroérosion à fil. L'examen des raisons invoquées par des cadres de l'Éducation locale pour justifier l'abandon de la voie habituelle ${ }^{6}$, aboutit au même type d'interprétation déjà proposée pour la corégulation formationemploi. La consolidation du mouvement pour la Vallée garantissait une croissance industrielle soutenue et donc la sécurisation des ressources de l'Éducation locale. Mais la dynamique se heurtait aux carences des PME en matière de stratégie de croissance. Les instances de formation se sont de ce fait mobilisées pour éliminer ces obstacles.

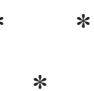

Parmi les chercheurs, un consensus existe selon lequel il n'existerait pas une «recette» universelle pour initier et développer un cluster industriel (Raines, 2002). Les cas de réussite ne seraient pas transférables car ils reposent sur des configurations locales spécifiques et sur un cheminement difficilement reproductible. Un modèle global n'existant pas, la recherche sur les clusters a en revanche apporté ou emprunté une multitude de modèles partiels. Ceux-ci décrivent des rouages appartenant à la dynamique d'ensemble du système productif local. Un modèletype particulier n'est pas intégré dans tous les systèmes, mais il est répertorié car observé dans de nombreux cas.

La recherche a élaboré notamment une catégorie de modèles portant sur le démarrage et l'expansion des actions communes. Les modèles correspondants soulignent l'importance de certains rôles, stratégies et dynamiques. La prégnance de ces structures ressort du fait qu'elles accroissent la certitude de retour sur investissement participatif. C'est dans cette catégorie de modèles que nous proposons de classer l'interaction cluster-formation.

Comme tout modèle qui prétend à une pertinence empirique, l'interaction cluster-formation est liée à

${ }^{6}$ La Skymill par exemple devait être «... une vitrine pour convaincre les jeunes », «... un équipement original pour singulariser l'IUT dans le paysage universitaire et industriel », «... un moyen pour démontrer aux entreprises de la région les bénéfices de l'UTGV ». 
des conditions préalables. Nous en définissons deux ci-dessous, le consensus des acteurs locaux pour l'objectif cluster étant donné comme acquis. Les deux conditions mises en exergue découlent des analyses d'Oliver et alii (1985) portant sur les dynamiques de l'action collective. Ainsi, une première condition concerne l'apparition d'un groupe qui se constitue en " masse critique ». Il s'agit d'un groupe d'acteurs suffisamment intéressés et équipés pour démarrer ou prolonger le projet à vocation collective. La rareté des groupes qui acquièrent ce statut s'explique entre autres par le découplage fréquent entre intérêts et ressources : les plus intéressés sont souvent démunis de ressources, et les plus pourvus en ressources sont souvent désintéressés. Une deuxième condition peut être définie comme la constitution des pionniers «en clique », c'est-à-dire soudés par de forts liens de loyauté. Ce type de réseau social est nécessaire pour « immuniser » l'avant-garde (la première masse critique) vis-à-vis des contrepressions, comme par exemple la résistance, l'échec, l'incertitude et la désertion. La Mecanic Vallée est donc un cas privilégié car l'amorce et l'évolution de ce projet bénéficiaient de l'émergence d'une masse critique et de la complicité des pionniers.

Les interactions constatées dans la Vallée ne constituent pas des processus isolés. Notre contribution a montré qu'ils sont en connexions, formant une réaction en chaîne et impliquant un nombre croissant de groupes : à l'alliance entre la firme Ratier et la municipalité de Figeac adhéraient successivement la Table Ronde des entreprises «marraines», les acteurs institutionnels des départements voisins (chambres consulaires, agences de développement, collectivités territoriales), puis les firmes plus «attentistes», et enfin les acteurs locaux de l'Éducation nationale.

Selon les analyses d'Oliver et alii (op. cit.), la réaction en chaîne concerne une population hétérogène, structurée en groupes caractérisés par des niveaux d'intérêts et de ressources divers. En fonction de ces vecteurs, les groupes ont des exigences différentes par rapport à la certitude en matière de résultat. Une cascade de participations se développe si les acquis réalisés dans une séquence sont suffisants pour atteindre le seuil d'exigence d'un groupe plus réservé.

L'influence de l'hétérogénéité des groupes sur le développement des participations explique pourquoi un succès local n'est guère reproductible. Une interaction isolée peut certes se dérouler dans des contextes variés, mais une série d'interactions, basée sur l'implication d'une diversité de groupes, s'enraye aisément en raison des particularités de l'hétérogénéité locale.

\section{Bibliographie}

Amin A., Thrift N. (1994), «Living in the Globa », in Amin A., Thrift N. (Eds.), Globalization, institutions and regional development in Europe, Oxford University Press, Oxford, pp. 1-22.

Blanc Ch. (2004), Pour un écosystème de la croissance, rapport au Premier ministre, Paris.

CNER, Conseil national des économies régionales (2001), «Donneurs d'ordres, sous-traitants et territoires », Inter Régions, numéro spécial 2001.

Coleman J. (1990), Foundations of Social Theory. 2 volumes, Cambridge University Press, Cambridge/ Massachusetts.
Combes M.-C., Quenson E. (2002), « Évaluation des baccalauréats professionnels de la productique », CPC-Documents, nº 2002.

Courault B. (2000), «Districts italiens et PMEsystèmes français - comparaison n'est pas raison ", La Lettre du CEE, $\mathrm{n}^{\circ}$ 62, février.

Dauty F. (2003), Réactivité industrielle, réseaux et territoires: quel modèle?, in SASE, «Éducation, connaissances et sociétés futures », 15th Annual Meeting on Socio-Economics, Aix en Provence, 2628 juin. 
Granovetter M. (1978), «Threshold Models of Collective Behavior », American Journal of Sociology, Vol. 83, n 6 , pp. 1420-1443.

Guillaume R. (coord.) (2003), Les SPL en MidiPyrénées: vers l'émergence de systèmes régionaux?, rapport final, programme CCRRDT Midi-Pyrénées, Toulouse, novembre.

Jobert A. (2000), «Les acteurs locaux face à la formation professionnelle », in Tallard M., Thérét B., Uri D., Innovations institutionnelles et territoires, L'Harmattan, Paris, pp. 239-262.

Lorenz E. (1988), « Neither Friends nor Strangers », in Gambetta D. (Ed.), Trust: Ma-king and Breaking Cooperative Relations, Oxford University Press, Oxford, pp. 194-210.

Macy M.W. (1991), "Chains of Cooperation: Threshold Effects in Collective Action », American Sociological Review, Vol. 56, n 6, pp. 730-747.

Maskell P., Malmberg A. (1999), « Localised Learning and Industrial Competitiveness », Cambridge Journal of Economics, Vol. 23, n² 2, pp. 167-185.

Mohrman S.A. (2003), «The Role of Networks in Fundamental Organizational Change ", Journal of Applied Behavioral Science, Vol. 39, n 3, pp. 301-323.
Oliver P.E., Marwell G., Teixeira R. (1985), «A Theory of the Critical Mass. Part 1 - Interdependence, Group Heterogeneity, and the Production of Collective Action ", American Journal of Sociology, Vol. 91, n 3, pp. 522-556.

Olson M. (1968) (1987, traduction), Logique de l'action collective, PUF, $200 \mathrm{p}$.

Pommier P. (2005), SPL et Pôles de compétitivité, Manuscrit, contribution à la session de la DIACT du 23/08/2005.

Porter M.E. (1998), On Competition, Harvard Business School Press, Boston.

Raines Ph. (Ed.) (2002), Cluster Development and Policy, Ashgate Publishing, Aldershot.

Raveyre M.-F., Saglio J. (1984), «Les systèmes industriels localisés: éléments pour une analyse sociologique des ensembles de PME industriels », Sociologie du travail n ${ }^{\circ}$ 2, pp. 157-176.

Rosenfeld S.A. (2000), "Community College/ Cluster Connections: Specialization and Competitiveness in the United States and Europe », Economic Development Quarterly, Vol. 14, n 1, pp. 51-62.

Veltz P. (2002), «Vingt ans de transformations de l'économie française ", Cahiers français $\mathrm{n}^{\circ} 311$, pp. 26-31.

\section{Résumé \\ "Mecanic vallée »: interactions entre système productif local et formation \\ Joachim Haas}

Cet article étudie le phénomène de renforcement interactif de la politique de formation et du système productif local. II s'intéresse plus particulièrement aux mécanismes concourant au développement des coopérations interentreprises et entre les sphères publique et privée locales. Le processus de renforcement mis en exergue suit le principe de la boucle récursive. Selon ce modèle, la progression des coopérations induit des investissements dans le domaine de la formation locale, et inversement, les investissements en matière d'infrastructures de formation font progresser les coopérations. Le projet de système productif local « Mecanic Vallée » est exemplaire à cet égard.

Mots-clés

Politique de formation, région, système productif, localisation d'entreprise, étude de cas Journal of Economic Literature: R 58, M 53, R 3 
